\title{
BMJ Open Protocol for a young adult mental health (Uspace) cohort: personalising multidimensional care in young people admitted to hospital
}

\author{
Ashleigh M Tickell (D , ${ }^{1}$ Cathrin Rohleder (D) , ${ }^{1}$ Alexandra Garland, ${ }^{1}$ \\ Yun Ju Christine Song, ${ }^{1}$ Joanne Sarah Carpenter (D) , ${ }^{1}$ Kate Harel, ${ }^{2}$ Lisa Parker, ${ }^{2}$ \\ lan B Hickie (D) , ${ }^{1}$ Elizabeth Scott (i) ${ }^{1,2}$
}

To cite: Tickell AM, Rohleder C, Garland $A$, et al. Protocol for a young adult mental health (Uspace) cohort: personalising multidimensional care in young people admitted to hospital. BMJ Open 2021;11:e038787. doi:10.1136/ bmjopen-2020-038787

- Prepublication history for this paper is available online. To view these files, please visit the journal online (http://dx.doi org/10.1136/bmjopen-2020038787).

Received 24 March 2020 Revised 10 0ctober 2020 Accepted 02 November 2020

Check for updates

(C) Author(s) (or their employer(s)) 2021. Re-use permitted under CC BY-NC. No commercial re-use. See rights and permissions. Published by BMJ.

${ }^{1}$ Brain and Mind Centre, The University of Sydney, Sydney, New South Wales, Australia

${ }^{2}$ Young Adult Mental Health Unit, St Vincent's Private Hospital, Darlinghurst, New South Wales, Australia

Correspondence to

Dr Ashleigh M Tickell;

ashleigh.tickell@sydney.edu.au

\section{ABSTRACT}

Introduction Currently, the literature on personalised and measurement-based mental healthcare is inadequate with major gaps in the development and evaluation of 21st century service models. Clinical presentations of mental ill health in young people are heterogeneous, and clinical and functional outcomes are often suboptimal. Thus, treatments provided in a person-centred and responsive fashion are critical to meet the unique needs of young people and improve individual outcomes. Personalised care also requires concurrent assessment of factors relating to outcomes and underlying neurobiology. This study builds on a completed feasibility study and will be the first to incorporate clinical, cognitive, circadian, metabolic and hormonal profiling with personalised and measurement-based care in a cohort of young people admitted to hospital.

Methods and analysis This prospective, transdiagnostic, observational study will be offered to all young people between the ages of 16 and 30 years admitted to the inpatient unit of the participating centre. In total, 400 participants will be recruited. On admission to hospital, young people will undergo clinical and diagnostic assessment, cognitive testing, self-report questionnaires, metabolic and hormonal data collection, and anthropomorphic measurements. Participants will wear an actigraphy watch for at least 1 week during admission to measure circadian patterns and sleepwake cycles. A feedback session between clinician and participant will occur after clinical and other laboratory assessments to tailor individual treatment plans, explain the ongoing process of measurement-based care, and provide participant and family education. Associations between cognitive impairments, disturbed sleep-wake behaviours, circadian rhythms, clinical symptoms and functional impairments will be evaluated to improve the understanding of parameters affecting clinical outcomes.

Ethics and dissemination This study protocol was approved by the Human Research Ethics Committees of the University of Sydney (HREC USYD 2015/867) and St Vincent's Hospital (HREC SVH 17/045). This study will be published on completion in a peer-reviewed journal.

\section{Strengths and limitations of this study}

- This study will follow a large cohort $(n=400)$ of young people admitted to hospital for mental health care to examine determinants of clinically significant mental and physical health, and social, educational and occupational outcomes.

- As an observational study this will be naturalistic in fashion and no randomisation or control group will be included.

- The multifaceted variables collected such as anthropomorphic, metabolic, hormonal, cognitive, circadian and symptomatic data will offer information on the multifactorial variables leading to mental and physical ill health and treatment outcomes.

- Heterogeneity of the clinical presentations to an inpatient setting strengthens the study's generalisability to other similarly severe presentations of mental ill health in young people.

\section{INTRODUCTION}

Clinical presentations of mental ill health in young people are very heterogeneous in fashion. Current diagnostic criteria often fit poorly with the subthreshold or mixed presentations that are common in young people. ${ }^{1}$ Typically, mental healthcare has relied on these diagnostic criteria to inform clinical decisions, leading to stereotyped and limited treatment responses, which are often not well matched to individual needs. In accordance with a shift in healthcare services and research priorities from evidence-based to personalised medicine, ${ }^{2}$ sequenced, multidisciplinary and more measurement-based interventions are new goals. These aim to translate evidence-based methodologies into individual treatment plans. ${ }^{2} 3$ Consequently, we have proposed a highly personalised and measurement-based care model for use in youth mental health services. ${ }^{4}$ This 
transdiagnostic, person-centred model of care emphasises early access to personalised care through a comprehensive multidimensional assessment of various clinical and functional domains, ${ }^{5}$ including social and occupational functioning, self-harm and suicide risk, alcohol and substance misuse, physical health, clinical severity and stage (stage 1a (non-specific symptoms) to stage 4 (severe, persistent and unremitting illness) $)^{67}$ and illness subtypes (psychosis, anxious depression, bipolar spectrum). It also links with putative pathophysiological pathways (neurodevelopmental, hyperarousal, circadian) as well as individual illness trajectories. ${ }^{18-10}$ Such a clinical stage-appropriate and transdiagnostic framework may lead to improved clinical outcomes as it helps to guide the selection of treatments based on individual needs and symptom burden as well as underlying pathophysiological mechanisms. ${ }^{1}$

\section{Objectives of the study and conceptual framework}

The 'Personalised Multidimensional Care' study will generate multidimensional-that is, clinical, functional, cognitive, circadian, metabolic and hormonal-profiles for participants with clinician feedback, specifically addressing individual issues affecting participant outcomes such as cognitive impairments, disturbed sleepwake behaviours and circadian rhythms, clinical symptoms and functional impairments.

The objectives of the study are:

- To establish a standardised measurement-based and personalised care research protocol in an inpatient unit.

- To evaluate clinical parameters that impact participant outcomes such as cognitive impairments, disturbed sleep-wake behaviours and circadian rhythms, clinical symptoms and functional impairments.

- To investigate associations between metabolic, hormonal, clinical, self-report and circadian factors.

- To compare the inpatient cohort data with similar data from young people presenting to outpatient youth mental health services (eg, Brain and Mind Centre cohorts ${ }^{911-13}$ ).

Cognitive testing and circadian profiling is not yet often routinely available in standard clinical care but is very important in understanding the relationship between symptoms and functioning, as neuropsychological deficits and dysregulated sleep-wake cycles have been linked to poorer functional outcomes. ${ }^{814-17}$

Furthermore, incorporation of metabolic and hormonal profiles is imperative in capturing, evaluating and treating comorbid and contributing conditions such as metabolic syndrome and polycystic ovarian syndrome (PCOS) which are often found concurrently in young psychiatric patients. ${ }^{18-21}$

The 'Personalised Multidimensional Care' study focuses on an inpatient young adult mental health clinic (Uspace). A key strength of this cohort is in its heterogeneity and ability to capture a presently severe hospitalised sample of young adults with varying mental disorders.
The cohort will allow for studying a wide range of personalised interventions, while also examining the longitudinal trajectory of this young, more severely ill cohort, and the associations between detailed clinical, hormonal and metabolic profiles, social, neurocognitive, functional and circadian outcomes.

As previously demonstrated, the use of Health Information Technologies (HITs) (computerised, self-reported e-health technologies such as the InnoWell Platform (Project Synergy, InnoWell) ${ }^{22}$ ) facilitates systematic multidimensional assessment in young people seeking help, ${ }^{23}$ enables opportune and timely responses from service providers and clinicians, ${ }^{23}$ and will, therefore, facilitate the development and delivery of highly personalised and measurement-based treatment plans.

The multidimensional assessments (clinical, functional, cognitive, circadian, metabolic and hormonal) and resulting personalised interventions will enhance knowledge and inform clinical practice to improve standard clinical care in an inpatient unit. However, personalised care means implementation of new protocols, training, and practice, as well as cultivating a new conceptual framework. ${ }^{24}{ }^{25}$ We have previously shown that it is feasible to integrate technology and neurocognitive testing as standard clinical care in an inpatient unit, and that neurocognitive profiling may help us to better understand the illness severity in young patients. ${ }^{24}$ Based on these results, we have decided to add further routine assessments (eg, circadian, metabolic and hormonal profiling) to be able to routinely provide personalised and measurement-based care. This study will integrate these approaches to help further knowledge in the field and facilitate translational changes to mental healthcare. Importantly, the personalised care approach based on multidimensional assessments is a collaborative approach and requires multidisciplinary teams of health professionals and involves shared decision-making based on the participant's best interests. ${ }^{25} 26$

\section{METHODS AND ANALYSIS}

\section{Study design and setting}

This prospective, transdiagnostic, observational study will offer recruitment to all inpatients aged 16-30 admitted to the Young Adult Mental Health Unit (Uspace), St Vincent's Private Hospital Sydney, Australia. Uspace is a voluntary private mental health service, targeted to the needs of young adults. Furthermore, it is a unique multidisciplinary clinical setting for research. Patients are referred for the assessment of mental health problems; with a mission to promote recovery and psychological well-being of young adults with severe and emerging mental health problems.

This 'Personalised Multidimensional Care' study has started in March 2020 and will be conducted over a period of 5 years with assessments at baseline and possible longitudinal follow-up available on participant consent up to 24 months (estimated end date of recruitment: March 
2023; estimated date of study completion: March 2025). That is, with the patient's consent, we will invite patients back for a follow-up assessment. At 24 months, the assessments conducted at baseline will be repeated. This will inform a unique tracking of the patients from an inpatient facility. We are in the process of an ethics amendment to include an additional 6-month and 12-month follow-up to improve the care provided as we have found this to be effective in previous experience with other studies.

The recruitment will be based on the presentation to care and is not restricted by specific diagnostic criteria. The diagnosis-independent selection of participants as well as the comprehensive assessments are consistent with the transdiagnostic 'Research Domain Criteria' (RDoC) approach. ${ }^{27}$ RDoC aims to classify mental disorders based on neurobiological and behavioural measures that cut across current disorder categories. The use of functional, circadian, hormonal, metabolic and cognitive assessments, allows to attain a comprehensive picture of the individuals admitted to USpace, and a subsequent classification based on biobehavioral dimensions. Furthermore, as this study is offered young people admitted to Uspace independent from diagnosis (ie, we will not exclude young people with a primary diagnosis outside a target category, a comorbid disorder or those who have some, but not all of the criteria required for a diagnosis of a specific disorder), our resulting cohort will have the appropriate variance as advocated by proponents of the RDoC approach. ${ }^{28}$ However, the vast majority of young people admitted to USpace will have a primary diagnosis of an affective disorder. These include depressive disorder, anxiety disorder, bipolar disorder or affective psychosis.

\section{Participant and lived experience involvement}

The HIT system (InnoWell Platform (InnoWell), ${ }^{22}$ which will be used for the collection of self-report data in the study, has been developed with participant and public involvement. Although young people were consulted during the development of the technology used to measure relevant outcomes of the study, they were not invited to comment on the study design. However, we will invite patient and lived experience involvement representatives to help us writing a plain language summary of the results and developing our dissemination strategy.

\section{Patient cohort}

Study participants are currently admitted young adult inpatients, aged 16-30, presenting with a severe affective episode (ie, depressive, manic, anxious; including those with psychotic features). Diagnosis will be a consensus diagnosis; that is, via multidisciplinary clinical assessment (by psychiatrists, psychologists and allied health professionals).

Exclusion criteria for this study are: (1) insufficient fluency in the English language to participate in the cognitive testing; (2) inability to consent due to intellectual impairment (for example, $\mathrm{IQ}<70$ as determined by the treating psychiatrist/psychologist) or severity of mental illness (as determined by the treating psychiatrist/psychologist) and (3) refusal to provide informed consent.

Comorbid or pre-existing childhood-onset conditions (eg, attention-deficit/hyperactivity disorder and conduct disorder), as well as alcohol or other substance misuse or autistic spectrum disorders are not exclusion criteria.

\section{Study course and procedures}

Potential participants who are admitted to USpace and interested in participating will be referred to the research study at arms-length to ensure that there is minimal perceived coercion from the referring clinician to the participant to participate in the study. The participant will then be in-depth informed by the research staff based in the clinic to introduce the study and undertake consent. Through consecutive referral, eligible participants who are newly admitted to Uspace will be booked in to complete neurocognitive testing and self-report questionnaires after informed written consent. Participants' availability and booking schedule is based around their own routine appointments and groups as inpatients, with most participants being booked within 4 days of admission. Hormonal and metabolic measures are routinely collected at Uspace on admission as part of standard clinical care, this data will also be used for research purposes on and during admission as indicated by standard of care, regardless of entry into the study. Cambridge Neuropsychological Test Automated Battery (CANTAB) is completed on a tablet devide, taking between 40 and $50 \mathrm{~min}$. The self-report questionnaire will be completed the day after neurocognitive testing, taking approximately $45-60 \mathrm{~min}$. Both, neurocognitive testing and the self-report questionnaire can be split and completed on two consecutive days. Actigraphy watches will be set up to record and given to patients to wear once informed consent is provided. Participants will wear the actigraphy watches during the admission period for a maximum of 14 days and a minimum of 6 days. No supervision is needed during wearing of the actiwatches. The appropriate clinician that admitted the corresponding participant during the admission process will complete a 'Clinical Assessment Form'. The multidimensional assessments as well as the feedback session will inform personalised interventions offered to the young adult participants in a shared decision-making process.

At follow-up, all assessments are repeated except for clinical assessment, as we expect that the follow-up assessments will be conducted after discharge in almost all cases. Therefore, the clinical assessment is only done in participants readmitted to USpace within the follow-up period.

An overview and timeline of the assessments is provided in table 1. 


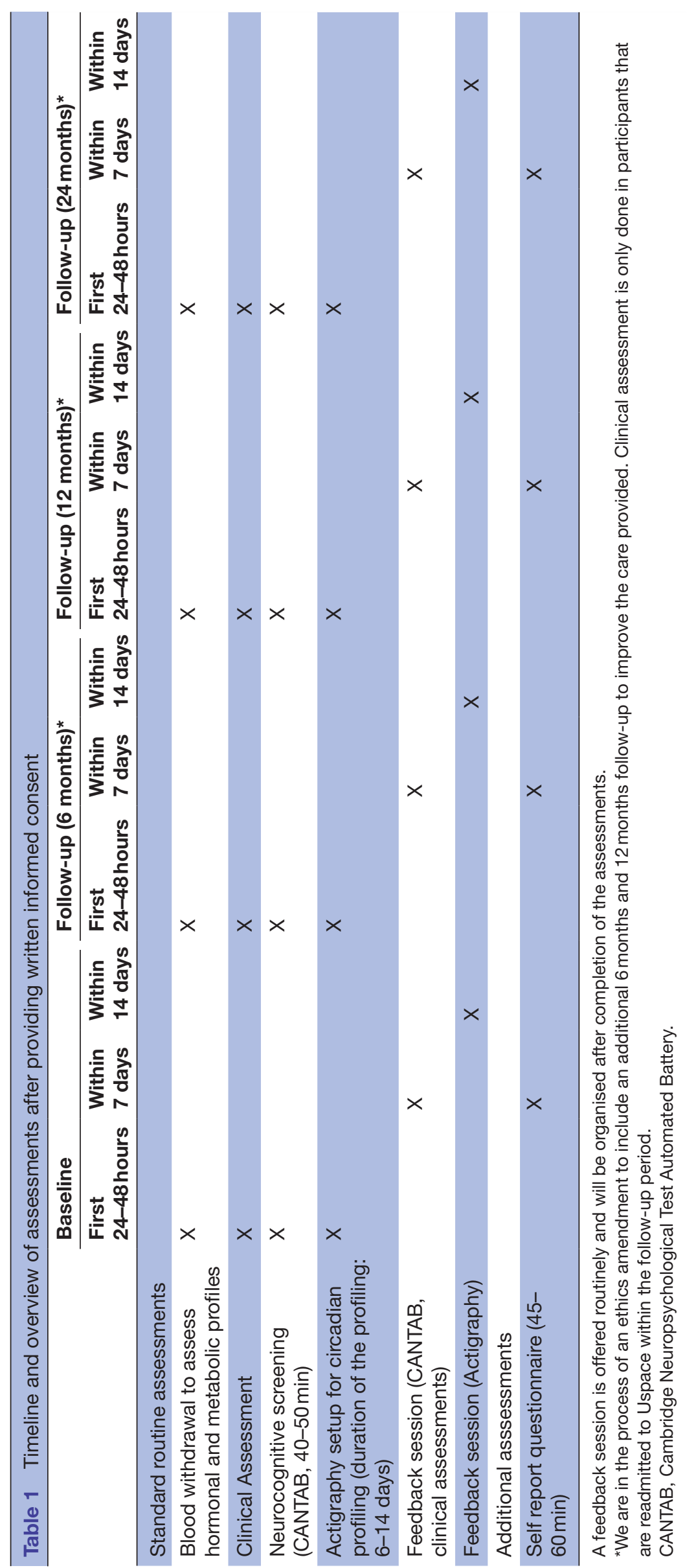

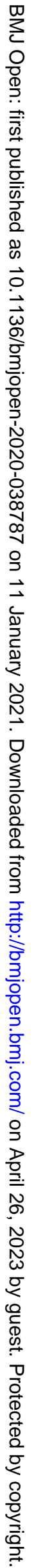




\section{Assessments}

\section{Clinical Presentation}

The clinical presentation is captured in the 'Clinical Assessment Form'. In addition, this form records information regarding the participants current episode/ presentation, psychiatric history, medical history, social and occupational functioning, clinical severity/improvement and clinical stage.

In addition, menstrual history and symptoms are assessed in the female population using a self-report questionnaire capturing age of menarche, regularity of the period, amenorrhoea, period pain, body hair, skin, mood, sleep and fatigue symptoms.

\section{Self-report questionnaire}

The self-report questionnaire is implemented within HIT system (InnoWell Platform (InnoWell) ${ }^{22}$ and is, thus, electronically completed on a touchscreen device (eg, tablet device). It captures key clinical information regarding the following:

\section{Demographics}

Biological sex is specified, and age is calculated. Current engagement in part-time or full-time education or employment is recorded to determine Not in Education, Employment or Training (NEET) status. NEET is assigned if there is no full-time or part-time education, employment, training or volunteer work. Current receipt of any government benefits is also recorded.

\section{Personal mental illness history}

Known childhood-onset disorders (ie, with clear onset prior to 12 years old) are recorded in addition to current diagnoses.

\section{Family history of mental illness}

Known family history of mental illness in first degree relatives is recorded.

\section{Treatment utilisation/history}

Exposure to classes of medication (antidepressant, antipsychotic, mood stabiliser or stimulant medication), and hospitalisation overnight or longer due to a mental health problem (including specification of hospitalisation due to illness severity or suicidality) are recorded.

\section{Alcohol and substance use}

The presence of any reported use of tobacco, alcohol, cannabis, stimulants or other drugs is recorded.

\section{Clinical symptoms}

Initial questions obtain key demographic and clinical information, focusing on critical illness course variables (eg, onset of symptoms, hospitalisations, age of first help seeking). Standardised questionnaires include the 10-item Kessler Psychological Distress Scale ${ }^{29}$ to detect psychological distress, with scores ranging between 10 and 50 (a score over 30 representing a likely severe mental disorder); Quick Inventory of Depressive Symptomatology- $16^{30}$ to assess severity of depressive symptoms, with scores ranging between 1 and 27 (a higher score representing greater severity of depression); Overall Anxiety Severity and Impairment Scale, a 5-item measure for assessment of severity and impairment in regard to anxiety symptoms, ${ }^{31}$ with scores ranging between 0 and 20 (a higher score representing a higher frequency and severity of anxiety (across anxiety disorders); Psychosis Screener derived from Community Assessment of Psychic Experiences, ${ }^{32}$ a positive symptoms scale and psychosis screener, developed to measure the lifetime prevalence of psychoticlike experiences in the general population; Hypomania Screener derived from the Altman Self-Rating Mania Scale, ${ }^{33}$ a 5-item self-rating scale to assess the severity of manic symptoms, a higher score (five or more) indicating a high probability of a manic or hypomanic condition; Suicidal Ideation Attributes Scale, ${ }^{34}$ a 5 -item scale to screen participants for suicidal thoughts and severity of these thoughts, with scores ranging between 0 and 50 (a higher score representing more severe suicidal thoughts (a score over 21 being in the high risk category); and, the Somatic and Psychological Health Report-12, ${ }^{35}$ a 12-item measure to screen for current depression and/or anxietylike symptoms (a score of two or more on the psychological subscale and a score of three or more on the somatic subscale indicating current depression and/or anxietylike symptoms).

\section{Neurocognitive screening}

Self-reported, non-structured, standardised questions in regard to the participant's own sense of cognition will be assessed prior to testing (eg, changes in everyday thinking skills and neurocognitive abilities).

A trained research psychologist or psychiatric registrar at Uspace will administer the cognitive testing battery, which includes computerised assessments. First, premorbid intellectual functioning ('predicted IQ') is estimated on the basis of performance on the Wechsler Test of Adult Reading. ${ }^{36}$ Following this, participants complete tests from the CANTAB ${ }^{37}$ on an HIT platform. CANTAB tests have the advantage of being largely nonverbal (ie, language-independent, culture-free) and have been described in detail elsewhere. ${ }^{37-39}$ Five tasks are included in this study: the Motor Screening Task, an introductory task to prepare participants for testing (ie, not included in overall results) using induction of sensorimotor and comprehension; the Verbal Recognition Memory task (immediate and recall/delayed) assessing 'verbal memory and new learning' indexed by the encoding and subsequent retrieval of verbal information scores; the Attention Switching Task assessing 'mental flexibility' and indexed by the total adjusted score; the Paired Association Learning task assessing 'visuospatial learning and memory' indexed by the total adjusted errors score from; and, the Rapid Visual Processing task (RVP) assessing 'sustained attention' and indexed by the RVP A prime (sensitivity to the target). 
Participants' individual, normed results are calculated by a trained research psychologist within 2-3 days of completion of cognitive testing battery. Calculation of CANTAB z-scores are completed for each participant. While each participant's predicted IQ is assessed in the cognitive screener, this is specifically to personalise results based on each participant's age, education and background, compared with 'demographically corrected' standardised scores (z-scores) using an internal normative database of healthy controls (http://www.camocog. com).

\section{Sleep-wake cycle/circadian profile}

Participants are given an actigraphy watch (GENEActiv watch, Activinsights, Kimbolton, UK) to wear on the non-dominant wrist during the admission period for a maximum of 14 days and a minimum of 6 days. This actigraph records motor activity levels, skin temperature and ambient light exposure continuously at a rate of $30 \mathrm{~Hz}$ for the duration of the recording period.

The GENEActiv devices have been validated against several types of accelerometry-based activity monitors ${ }^{40-42}$ as well as for sleep-wake scoring. ${ }^{43}$ More specifically, sleepwake detection in data collected with the GENEActiv devices has been found to have strong reliability and validity when compared with data collected with other GENEActiv monitors or with Actiwatch Spectrum devices (a well-established instrument in sleep and circadian research).$^{43}$ For over three decades, actigraphy monitors like the GENEActiv devices have been considered as noninvasive instruments to measure sleep and activity patterns and have been used extensively for research purposes in diverse clinical settings including: sleep disorders, various medical illnesses (eg, cancer, HIV, traumatic brain injuries, neurodegenerative diseases) and mental disorders (eg, anxiety, depression, bipolar and psychotic disorders).

\section{Metabolic and hormonal profiles}

As standard clinical care, metabolic data, including measures of body, weight, waist circumference, as well as blood pressure readings and routine metabolic bloods (including for example, glucose, insulin, cholesterol, low-density and high-density lipoprotein, triglycerides, etc) are routinely collected at Uspace on admission, this data will be used for research purposes. After assessment by a clinician, women with clinical evidence of comorbid PCOS will undergo further hormonal assays (including, eg, oestradiol, dehydroepiandrosterone sulfate, antimullerian hormone) collected as standard clinical care.

\section{Feedback and personalised care}

Once both the self-report questionnaire and cognitive testing have been completed by the participant, and the clinical assessment form has been completed by the admitting clinician, a feedback report will be generated (within 7 days of the completed assessments). A brief feedback session will be organised with the participant in order to go through and explain results and what that means in terms of more personalised intervention. This feedback report and session will be between the participant, treating clinician and clinical researcher. The feedback session will be part of the standard clinical care whereby the results of the testing will be explained in detail to the participant, management options will be explored, and any questions the participant may have will be addressed; furthermore, briefing will occur during the participant's weekly clinical reviews. The feedback session aims to give the participant a better understanding of certain aspects of their mental health and well-being and what they, and their clinicians, can do to improve those areas. A feedback report of the actigraphy (sleep-wake cycle and circadian) assessment results will be provided to the clinician on completion of the total time (maximum 14 days) wearing the actigraphy watch.

The results of the multidimensional assessmentsincluding clinical, functional, cognitive, circadian, metabolic and hormonal profiles-as well as the feedback session will inform personalised interventions offered to the young adult participant in a shared decision-making process.

\section{Sample size calculation}

We aim to include 400 participants annually, based on our knowledge typically 700-800 inpatients are admitted to Uspace per year. Thus, we expect to be able to collect data from 2000 participants over the period of the study. Although patient retention in youth mental health services is difficult to predict, ${ }^{44}$ in our experience, $70 \%$ of inpatients are retained from baseline and throughout follow-up assessments. This has been accounted for in our sample size estimation.

\section{Data analysis plan}

Once the participants have completed the self-report assessment, the data are collated and displayed as a detailed and immediate dashboard of results. This information is available to a trained research psychologist immediately on the participant's completion of the selfreport assessment and will be used to prepare the feedback report.

This 'Personalised Multidimensional Care' study allows for the assessment of multidimensional-that is, clinical, functional, cognitive, circadian, metabolic and hormonal-profiles for young adult participants. This includes:

- Evaluating parameters affecting participant outcomes such as cognitive impairments, disturbed sleep-wake behaviours and circadian rhythms, clinical symptoms and functional impairments.

- Investigating associations between metabolic, hormonal, clinical, self-report and circadian factors.

- Comparisons with similar data from young people presenting to outpatient youth mental health services (eg, Brain and Mind Centre cohorts ${ }^{911-13}$ ).

As the data collected will be highly multidimensional, aside from the use of standard statistical approaches (eg, 
analysis of variance, correlations, regression), we will employ more advanced statistical techniques to investigate the underlying interactions between demographics, clinical presentation, neurocognition, sleep-wake profiles and metabolics profiles in driving mental and physical ill health. These approaches include mixed-effects modelling as this is suited to data where samples are observed repeatedly, Bayesian modelling as this can be used to estimate the level of uncertainity in our parameter estimations, ${ }^{45} 46$ structural equation modelling ${ }^{47}$ and more data-driven techniques, ${ }^{4-50}$ such as hierarchical cluster analysis, ${ }^{1524} 51$ latent profile analysis ${ }^{52}$ and group-based trajectory modelling ${ }^{53}$ will be applied. To take advantage of the multidimensional and longitudinal nature of the data collected, machine learning approaches can be used to build models predictive, at baseline, of downstream physical and mental ill health outcomes. Algorithms that also provide some transparency in variable importance such as tree-based algorithms (Random Forest, XGBoost) and penalised regression (LASSO, Elastic-net) will be suitable for this.

To handle missing data points, we will also use (1) maximum likelihood approaches as these can estimate the most likely value of a parameter based on the observed data points and (2) multiple imputation to generate multiple imputed datasets where each dataset is analysed separately and the results are pooled. This approach ascertains the sensitivity of the statistical analysis based on different imputation estimates.

\section{ETHICS AND DISSEMINATION}

This study protocol was approved by the University of Sydney (HREC USYD 2015/867) and St Vincent's Hospital Human Research Ethics Committees (HREC SVH 17/045). Following confirmation that potential participants from the Uspace inpatient cohort are of mental and intellectual capacity to give informed consent, and have no language barriers, a complete description of the study will be discussed and eligible participants will be given a participant information sheet and consent form and followed up by the researcher for informed written consent, no less than 24 hours later, with those under the age of 18 having their parent or guardian also consent. This study is minimally invasive, and any adverse outcomes will inform procedure.

All participant data will be deidentified and stored in accordance with applicable security standards; therefore, the privacy of all participants will be protected.

The research findings will be disseminated through publications in peer-reviewed journals and conference proceedings, and participant data will be non-identifiable.

Contributors IBH and ES conceived the research idea, designed the study and are the principal investigators (for corresponding sites); AMT contributed to study conception, wrote the study protocol with input of JSC (actigraphy), AG (hormonal and metabolic measurements), KH, LP and ES. AMT manages the study with input of EMS, KH and LP. ES contributes to clinical organisation; KH and LP manage patient unit participation and supervision. AMT wrote the first draft of the manuscript; CR, AG and YJCS contributed to major edits in later versions. All authors contributed to discussion and have approved the final protocol manuscript.

Funding This study received philanthropic funding support (scholarship) donated by the Cottle Family to the Young Adult Mental Health Unit, St Vincent's Private Hospital and other philanthropic family donors who wish to remain anonymous.

Competing interests IBH was an inaugural Commissioner on Australia's National Mental Health Commission (2012-2018). He is the codirector, health and policy at the Brain and Mind Centre (BMC) University of Sydney. The BMC operates an earlyintervention youth services at Camperdown under contract to headspace. IBH has previously led communitybased and pharmaceutical industry-supported (Wyeth, Eli Lily, Servier, Pfizer, AstraZeneca) projects focused on the identification and better management of anxiety and depression. He was a member of the Medical Advisory Panel for Medibank Private until October 2017, a Board Member of Psychosis Australia Trust and a member of Veterans Mental Health Clinical Reference group. He is the chief scientific advisor to, and a $5 \%$ equity shareholder in, InnoWell. InnoWell was formed by the University of Sydney (45\% equity) and PwC (Australia; $45 \%$ equity) to deliver the \$A30 M Australian Government-funded Project Synergy (2017-2020; a 3-year program for the transformation of mental health services) and to lead transformation of mental health services internationally through the use of innovative technologies. ES is the Medical Director, Young Adult Mental Health Unit, St Vincent's Hospital Darlinghurst, Discipline Leader of Adult Mental Health, School of Medicine, University of Notre Dame, Research Affiliate, The University of Sydney and Consultant Psychiatrist. She has received honoraria for educational seminars related to the clinical management of depressive disorders supported by Servier and Eli-Lilly pharmaceuticals. She has participated in a national advisory board for the antidepressant compound Pristiq, manufactured by Pfizer. She was the National Coordinator of an antidepressant trial sponsored by Servier

Patient and public involvement Patients and/or the public were not involved in the design, or conduct, or reporting, or dissemination plans of this research.

Patient consent for publication Not required.

Provenance and peer review Not commissioned; externally peer reviewed.

Open access This is an open access article distributed in accordance with the Creative Commons Attribution Non Commercial (CC BY-NC 4.0) license, which permits others to distribute, remix, adapt, build upon this work non-commercially, and license their derivative works on different terms, provided the original work is properly cited, appropriate credit is given, any changes made indicated, and the use is non-commercial. See: http://creativecommons.org/licenses/by-nc/4.0/.

\section{ORCID iDs}

Ashleigh M Tickell http://orcid.org/0000-0002-8000-7864

Cathrin Rohleder http://orcid.org/0000-0002-3559-1846

Joanne Sarah Carpenter http://orcid.org/0000-0002-9766-6700

lan B Hickie http://orcid.org/0000-0001-8832-9895

Elizabeth Scott http://orcid.org/0000-0003-3907-0324

\section{REFERENCES}

1 Rohleder Cet al. Right care, first time: a highly personalised and measurement-based care model to manage youth mental health - Personalising care options in youth mental health: using multidimensional assessment, clinical stage, pathophysiological mechanisms, and individual illness trajectories to guide treatment selection. Medical Journal of Australia 2019;211:S32-41.

$2 \mathrm{Ng}$ MY, Weisz JR. Annual research review: building a science of personalized intervention for youth mental health. J Child Psychol Psychiatry 2016;57:216-36.

3 lorfino F, Hickie IB, Lee RSC, et al. The underlying neurobiology of key functional domains in young people with mood and anxiety disorders: a systematic review. BMC Psychiatry 2016;16:156.

4 Hickie IB, Scott EM, Cross SP, et al. Right care, first time: a highly personalised and measurement-based care model to manage youth mental health. Med J Aust 2019;211 Suppl 9:S3-46.

5 Hickie IB. Moving beyond stepped care to staged care using a novel, technology-enabled care model for youth mental health. Med J Aust 2019;211:404-5.

6 Hickie IB, Scott EM, Hermens DF, et al. Applying clinical staging to young people who present for mental health care. Early Interv Psychiatry 2013;7:31-43.

7 Scott J, Leboyer M, Hickie I, et al. Clinical staging in psychiatry: a cross-cutting model of diagnosis with heuristic and practical value. Br J Psychiatry 2013;202:243-5. 
8 Crouse JJet al. Right care, first time: a highly personalised and measurement-based care model to manage youth mental health - A comprehensive assessment framework for youth mental health: guiding highly personalised and measurement-based care using multidimensional and objective measures. Medical Journal of Australia 2019;211:S23-31.

9 Hickie IB, Frank lorfino JSC, Scott E, et al. The utility of clinical staging in youth mental health settings: neurobiological and longitudinal data from Sydney-Based studies of Transdiagnostic cohorts, in clinical staging in psychiatry: making diagnosis work for research and treatment. Cambridge: Cambridge University Press, 2019: 81-102.

10 Carpenter JSet al. Right care, first time: a highly personalised and measurement-based care model to manage youth mental health - Combining clinical stage and pathophysiological mechanisms to understand illness trajectories in young people with emerging mood and psychotic syndromes. Medical Journal of Australia 2019;211:S12-20.

11 lorfino F, Scott EM, Carpenter JS, et al. Clinical Stage Transitions in Persons Aged 12 to 25 Years Presenting to Early Intervention Mental Health Services With Anxiety, Mood, and Psychotic Disorders. JAMA Psychiatry 2019;76:1167.

12 Iorfino F, Hermens DF, Cross Shane, PM, et al. Delineating the trajectories of social and occupational functioning of young people attending early intervention mental health services in Australia: a longitudinal study. BMJ Open 2018;8): :e020678.

13 Carpenter JS, lorfino F, Cross S, et al. Cohort profile: the Brain and Mind Centre Optymise cohort: tracking multidimensional outcomes in young people presenting for mental healthcare. BMJ Open 2020;10:e030985.

14 Scott EM, Robillard R, Hermens DF, et al. Dysregulated sleep-wake cycles in young people are associated with emerging stages of major mental disorders. Early Interv Psychiatry 2016;10:63-70.

15 Tickell AM, Scott EM, Davenport T, et al. Neurocognitive clusters: a pilot study of young people with affective disorders in an inpatient facility. J Affect Disord 2019;242:80-6.

16 Tickell AM, Lee RSC, Hickie IB, et al. The course of neuropsychological functioning in young people with attenuated vs discrete mental disorders. Early Interv Psychiatry 2019;13): :425-33.

17 Lee RSC, Hermens DF, Redoblado-Hodge MA, et al. Neuropsychological and socio-occupational functioning in young psychiatric outpatients: a longitudinal investigation. PLoS One 2013;8): : :58176.

18 Scott EM, Hermens DF, White D, et al. Body mass, cardiovascular risk and metabolic characteristics of young persons presenting for mental healthcare in Sydney, Australia. BMJ Open 2015;5:e007066.

19 Brutocao C, Zaiem F, Alsawas M, et al. Psychiatric disorders in women with polycystic ovary syndrome: a systematic review and meta-analysis. Endocrine 2018;62:318-25.

20 Harnod T, et al, Association between depression risk and polycystic ovarian syndrome in young women. A retrospective nationwide population-based cohort study (1998-2013). Hum Reprod 2019;34:1830-7.

21 Mottillo S, Filion KB, Genest J, et al. The metabolic syndrome and cardiovascular risk a systematic review and meta-analysis. J Am Coll Cardiol 2010;56:1113-32.

22 Iorfino F, Cross SP, Davenport T, et al. A digital platform designed for youth mental health services to deliver personalized and Measurement-Based care. Front Psychiatry 2019;10:595.

23 Iorfino F, Davenport TA, Ospina-Pinillos L, et al. Using new and emerging technologies to identify and respond to suicidality among help-seeking young people: a cross-sectional study. J Med Internet Res 2017;19:e247.

24 Tickell AM, Scott EM, Davenport T, et al. Developing neurocognitive standard clinical care: a study of young adult inpatients. Psychiatry Res 2019;276: :232-8.

25 Cross SPet al. Right care, first time: a highly personalised and measurement-based care model to manage youth mental health - A service delivery model to support highly personalised and measurement-based care in youth mental health. Medical Journal of Australia 2019;211:S42-6.

26 Tickell AM, Scott EM, Hickie IB, et al. A case study of feedback and cognitive assessment of a young adult inpatient with major depressive disorder. Australas Psychiatry 2019;27:302-6.

27 Cuthbert BN, Insel TR. Toward the future of psychiatric diagnosis: the seven pillars of RDoC. BMC Med 2013:11:126

28 Casey BJ, Craddock N, Cuthbert BN, et al. DSM-5 and RDoC: progress in psychiatry research? Nat Rev Neurosci 2013;14:810-4.
29 Kessler RC, Andrews G, Colpe LJ, et al. Short screening scales to monitor population prevalences and trends in non-specific psychological distress. Psychol Med 2002;32:959-76.

30 Rush AJ, Trivedi MH, Ibrahim HM, et al. The 16-Item quick inventory of depressive symptomatology (QIDS), clinician rating (QIDS-C), and self-report (QIDS-SR): a psychometric evaluation in patients with chronic major depression. Biol Psychiatry 2003;54:573-83.

31 Norman SB, Cissell SH, Means-Christensen AJ, et al. Development and validation of an overall anxiety severity and impairment scale (OASIS). Depress Anxiety 2006;23:245-9.

32 Stefanis NC, Hanssen M, Smirnis NK, et al. Evidence that three dimensions of psychosis have a distribution in the general population. Psychol Med 2002;32:347-58.

33 Altman EG, Hedeker D, Peterson JL, et al. The Altman self-rating mania scale. Biol Psychiatry 1997:42:948-55.

34 van Spijker BAJ, Batterham PJ, Calear AL, et al. The suicidal ideation attributes scale (SIDAS): community-based validation study of a new scale for the measurement of suicidal ideation. Suicide Life Threat Behav 2014;44:408-19.

35 Hickie IB, Davenport TA, Hadzi-Pavlovic D, et al. Development of a simple screening tool for common mental disorders in general practice. Med J Aust 2001;175:S10-17.

36 Wechsler D. Wechsler test of adult reading. San Antonio, Tx: Psychological Corporation, 2001.

37 Sahakian BJ, Owen AM. Computerized assessment in neuropsychiatry using CANTAB: discussion paper. J R Soc Med 1992;85:399-402.

38 Sweeney JA, Kmiec JA, Kupfer DJ. Neuropsychologic impairments in bipolar and unipolar mood disorders on the CANTAB neurocognitive battery. Biol Psychiatry 2000;48:674-84.

39 Hermens DF, Redoblado Hodge MA, Naismith SL, et al. Neuropsychological clustering highlights cognitive differences in young people presenting with depressive symptoms. $J$ Int Neuropsychol Soc 2011:17:267-76.

40 Schaefer CA, Nigg CR, Hill JO, et al. Establishing and evaluating wrist cutpoints for the GENEActiv accelerometer in youth. Med Sci Sports Exerc 2014;46:826-33.

41 Hildebrand M, VAN Hees VT, Hansen BH, et al. Age group comparability of raw accelerometer output from wrist- and hip-worn monitors. Med Sci Sports Exerc 2014;46): :1816-24.

42 Esliger DW, Rowlands AV, Hurst TL, et al. Validation of the GENEA Accelerometer. Med Sci Sports Exerc 2011;43:1085-93.

43 te Lindert BHW, Van Someren EJW. Sleep estimates using microelectromechanical systems (MEMS). Sleep 2013;36:781-9.

44 Miller LM, Southam-Gerow MA, Allin RB. Who stays in treatment? child and family predictors of youth client retention in a public mental health agency. Child Youth Care Forum 2008;37:153-70.

45 Lee RSC, Hermens DF, Scott J, et al. A transdiagnostic study of education, employment, and training outcomes in young people with mental illness. Psychol Med 2017;47:2061-70.

46 lorfino Fet al. Predictors of clinical stage transitions among young people presenting to early intervention services with anxiety, mood, or psychotic disorders. JAMA Psychiatry.

47 Lee RSC, Hermens DF, Naismith SL, et al. Clinical, neurocognitive and demographic factors associated with functional impairment in the Australian brain and mind youth cohort study (2008-2016). BMJ Open 2018;8:e022659.

48 Lee RSC, Hermens DF, Naismith SL, et al. Neuropsychological and functional outcomes in recent-onset major depression, bipolar disorder and schizophrenia-spectrum disorders: a longitudinal cohort study. Transl Psychiatry 2015:5:e555.

49 Hermens DF, Lagopoulos J, Naismith SL, et al. Distinct neurometabolic profiles are evident in the anterior cingulate of young people with major psychiatric disorders. Trans/ Psychiatry 2012;2:e110.

50 Hermens DF, Naismith SL, Chitty KM, et al. Cluster analysis reveals abnormal hippocampal neurometabolic profiles in young people with mood disorders. Eur Neuropsychopharmacol 2015;25:836-45.

51 Crouse JJ, Moustafa AA, Bogaty SER, et al. Parcellating cognitive heterogeneity in early psychosis-spectrum illnesses: a cluster analysis. Schizophr Res 2018;202:91-8.

52 Crouse JJ, Chitty KM, lorfino F, et al. Exploring associations between early substance use and longitudinal socio-occupational functioning in young people engaged in a mental health service. PLoS One 2019;14:e0210877.

53 lorfino F, Hermens DF, Cross SP, et al. Delineating the trajectories of social and occupational functioning of young people attending early intervention mental health services in Australia: a longitudinal study. BMJ Open 2018;8:e020678. 